\title{
Factors Associated with Home Births among Rural Mothers in Uganda; a De- scriptive Survey of Gomba District
}

\author{
Atuhaire $\mathrm{S}^{*}$ \\ Science Department, Kabulasoke Teachers College, Mpigi, Uganda
}

*Corresponding author: Atuhaire S, Science Department, Kabulasoke Teachers College, P.O Box: P O 91, Mpigi, Uganda, Tel: 0774636127, E-mail: atuhaireshina@yahoo.com

Citation: Atuhaire S (2019) Factors Associated with Home Births among Rural Mothers in Uganda; a Descriptive Survey of Gomba District. J Public Health Hygiene Safety 1(1): 103. doi: 10.15744/2767-8792.1.103

Received Date: January 17, 2019 Accepted Date: December 17, 2019 Published Date: December 19, 2019

\begin{abstract}
Background: In Uganda, about 16 mothers die daily due to pregnancy, childbirth complications or within the first six weeks after delivery. For each maternal death, at least six mothers sustain permanent or temporary disabilities and morbidities. Until now, nearly $42 \%$ of the women still deliver at home and yet, $94 \%$ of them attend antenatal care at least once. Factors for the persistence of home births among mothers in rural areas of Uganda were therefore investigated to specifically determine the association between social demographic characteristics and home births, the impact of village health teams and the role played by sexual partners in influencing home births.

Methods: Through a prospective descriptive survey, data were collected from 133 mothers and fathers using a questionnaire and focus group discussion while 6 key informants were interviewed. quantitative data were thematically analyzed while quantitative data was analyzed for descriptive statistics and chi-square to determine the association of social demographic characteristics and home births.

Results: Social demographic characteristics including age, parity, distance, level of education of both partners, and culture were associated with home births except religion, tribe and health facility characteristics. VHTs occasionally referred mothers for health facility birth and partners greatly influenced the choice of birthplace.

Conclusion: Bridging the distance between homes and health facilities as well as empowering the girl child through education would reduce home births. VHTs and partners should be lobbied to actively engage in maternal and childbirth.

Keywords: Home Births; Natural Childbirth; Skilled Birth Attendance; Traditional Birth Attendant

List of Abbreviations: ANC: Antenatal Care; HC: Health Center; HIV: Human Immuno-deficiency Virus; MOH: Ministry of Health; NGO: Non-Governmental Organizations; PMTCT: Prevention of Mother to Child Transmission; SBAs: Skilled Birth Attendants; SPSS: Statistical Package for social scientists; TBA: Traditional Birth Attendant; USAID: United States Agency for International Development; VHT Village Health Teams
\end{abstract}

\section{Introduction}

Home birth is the process of bringing forth a child by humans in their places of residence or in a non-clinical setting typically using natural childbirth methods [1,2]. Health facility birth assures safety and survival to the mother and her neonate and yet in developing countries, home births are rampant contributing to more than three-quarters of maternal deaths [4] and maternal complications during pregnancy, childbirth or within the first six weeks after childbirth [3-5]. The complications include postpartum hemorrhage, eclampsia, infections, and unsafe abortion [3,5,6]. Maternal death may also be due to indirect causes aggravated by pregnancy such as diabetes, malaria, anemia, and hepatitis [5]. Some mothers sustain chronic or permanent disabilities such as obstetric fistula while others may have stillborn babies or lose their newborn [6,7]. Such atrocities are actually preventable if mothers fully utilized maternal health care and if governments and partners ensured each facility had at least a midwife, vital equipment, basic medicine and clean birth environment [6].

Averagely in Sub-Saharan Africa, about 58\% of total births still occur in home settings and are either attended to by a Traditional Birth Attendant (TBA), a relative or the mother herself despite nearly $94 \%$ of them having attended antenatal care at least once $[5,8,9]$. In some specific regions of African and South Asian countries such as Nigeria, Ethiopia, Pakistan and Nepal, home births are as high as 70\%, 95.9\%, 74\%, and 69\% respectively and Zambia, 65\% [4,10]. Traditional birthing practices in a home setting indicate endurance and even in an event of maternal death, though sad, it is still considered a normal event [11]. In Ethiopia, nearly all women give birth at home with the exception of a few who develop complications such as prolonged obstructed labor and end up 
delivering in a health facility [12]. In communities where home births are very high, TBAs are also quite many and yet they lack skills of handling maternal and childbirth complications. They are not trained but have basically acquired skills in the process of delivering their own babies by themselves or through an apprenticeship and training by other TBAs [8]. This has in most instances resulted in intrapartum complications which contribute to 225,000 maternal deaths, 904000 neonatal deaths, and 1.02 million stillbirths that are experienced annually in the region [13].

The government of the Republic of Uganda is lauded for a reduction in maternal mortality ratio from 684 per 100,000 live births in 1995 to 343 deaths per 100,000 live births in 2015 [14]. She is also in partnership with several bilateral and multilateral stakeholders to build and ensure core capacities for public health concerns [14]. As a result, 94\% of the mothers receive Antenatal Care (ANC) services at least once, and about $79 \%$ and $58 \%$ of the mothers in urban and rural areas respectively deliver their babies in health facilities $[3,5,10,15]$. However, comparing urban and rural health facility birth, it is evident that health facility birth in rural areas are still low and this has contributed to higher maternal and child mortalities in rural areas than in urban areas $[3,5,16]$.

Maternal and perinatal mortality and morbidity levels are key indicators of public health in each country worldwide [3,5,6,10,14]. However, in low-income countries, such indicators are still desired and unfortunately, the burden is shouldered by poor and vulnerable groups of people residing in remote areas where skilled attendance at birth, family planning, and antenatal care interventions and utilisation are still low $[11,14,15,17]$. Additionally, the poor and vulnerable group are characterized by the three delays which should be eliminated and these include; a woman's decision to seek care, a woman's ability to reach a health care facility and a woman's ability to receive adequate care [13,17]. Much as a large number of women may be aware of the benefits of health facility delivery, lack of autonomy and their position in a household makes them delay to make a decision to have health facility delivery. As such, often they do not desire to go against family wishes and decisions. In some instances, they lack transportation funds and some generally are biased against public incentives which make them opt for home delivery [17]. This has a public health implication not only to individual women but also their families and health care system.

In an attempt to address public health challenges related to home births, the government of Uganda initiated Village Health Teams (VHTs) concept in 2001 as a national strategy to improve community health, link up the villages and the health system, work closely with local leaders to improve the health of pregnant women and their children through support and education, carry out home visits, together with families plan for emergency transportation of pregnant women during labor or during post-partum and neonate, engaging communities in planning for their own health and provision of interventions in form of primary health care, and mobilize community members for health interventions such as mass immunization, provide health education, and refer patients for facility-based services [18-20]. Despite various efforts by the government and stakeholders and the stipulated role of VHTs concerning pregnant women, presences of, midwives up to $\mathrm{HC}$ ii, and free delivery services in public facilities, home births in rural areas are still high and little is documented concerning probable factors. Therefore this study set out to determine factors associated with the persistence of home births in the rural area of Gomba where health facility births were low for the years 2011, 2012, and 2013 as indicated in the District Health Management System report 2011- 2013 summarized in Table 1, The study is conceptualized as in Figure 1 [21].

1) TC measurement

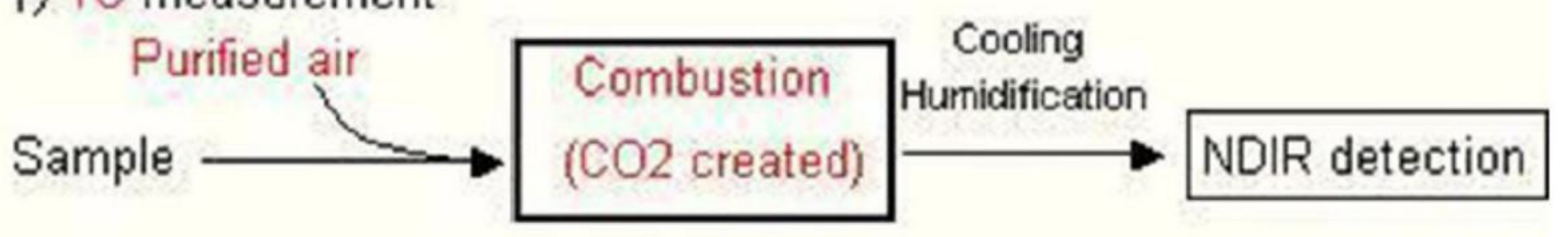

Figure 1: Conceptual Framework

\begin{tabular}{|c|c|c|c|c|r|r|r|r|r|r|r|r|}
\hline \multirow{2}{*}{ Year } & \multicolumn{10}{|c|}{ Meliveries in Health Units } \\
\cline { 2 - 15 } & \multicolumn{10}{|c|}{ Month } \\
\cline { 2 - 13 } & Jan & Feb & Mar & Apr & May & Jun & Jul & Aug & Sep & Oct & Nov & Dec \\
\hline 2011 & - & - & - & - & - & - & 76 & 126 & 112 & 136 & 119 & 118 \\
\hline $\mathbf{2 0 1 2}$ & 132 & 132 & 114 & 117 & 108 & 119 & 111 & 123 & 88 & 162 & 107 & 150 \\
\hline $\mathbf{2 0 1 3}$ & 138 & 148 & 167 & - & - & - & - & - & - & - & - & - \\
\hline
\end{tabular}

Table 1: Showing deliveries in health unit in Gomba district since 2011- 2013 Adopted from HMIS, Gomba district (2012)

The conceptual framework on home births was adopted and modified from Gwamaka S' (2012) study on utilization and factors affecting delivery in health facilities. The framework highlights that home births are influenced by factors including the women's 
social demographic characteristics, active involvement of their partners, functional of referral systems, and health facility characteristics.

\section{Materials and Methods}

\section{Study Location}

The study was conducted in Gomba district in the central region in Uganda. Uganda is located in East Africa between latitudes; $4^{\circ}$ north, $2^{\circ}$ south, $30^{\circ}$ west and $34^{\circ}$ east. It is composed of four administrative regions, 112 districts with 38.38 million people as per the 2014 national census [22]. The administrative regions include: northern, eastern, western and central regions. Gomba district is one of the newly formed districts and was curved from Mpigi district in July 2010. Before 2010 it was one of the counties of the greater Mpigi district. It has four sub-counties which include Mpenja, Kyegonza, Kabulasoke, Maddu and Kanoni town council where the district headquarters are located. It lies approximately 97 kilometers southwest of Kampala at coordinates; $0011 \mathrm{~N}$, 31 $55 \mathrm{E}$.

\section{Study Design and Population}

A prospective survey design was used to collect qualitative and quantitative data about women of reproductive age (15 to 49 years old) who had home births in the past two years before the study. A semi-structured questionnaire, focus group discussions and key informant interview were used to obtain required information from participants who included mothers, or their partners, health center iii administrators and midwives about the various factors associated with home births in the area.

The data obtained from the mothers who had health facilities births in 2012 in Gomba district was summarized in the districts' Health Management and Information Systems (HMIS) as shown in Table 1. The National Statistics of 2012, reported that the total population of Gomba district was152, 800 whereas the proportion of deliveries according to the Ministry of Health (MOH) was 0.05 percent of the entire population in the district per year which is equivalent to $0.05 \times 152800=7640$ women of reproductive age

Therefore, the expected number of deliveries in Gomba district for the financial year 2012/2013 was 7640 . However, 7640 was for the whole year. The monthly expected deliveries, assuming an equal distribution of births throughout the year were estimated at;

$\frac{7640}{12}=637$ Deliveries in the district per month

In reference to Table 1, the highest number of births in the health facilities, the district had ever attained was 167 (March 2013 ) [21].

Which is equivalent to $\frac{167 \times 100}{637}=26 \%$

This indicated that only 26\% (167) mothers were attended to by Skilled Birth Attendants (SBAs) and 74\% (470) mothers were not attended to by SBA during childbirth. Despite the low proportion of health facility births in the district, no study had been done to determine factors for the same.

\section{Sampling and Sample Size Determination}

Systematic random sampling was done from the sub-counties to parishes and then to villages. Two sub-counties (Mpenja and Kabulasoke) were randomly sampled from which four parishes (Bulwadda, and Butiti from Kabulasoke; and Bbinzi and Golola from Mpenja) were subsequently randomly selected. Thereafter eight villages; two from each parish that is to say; Bulwadda: Kateete A, and Kiziiko. Butiti: Kabulasoke A and Lubale. Binzi: Bbuye and Budonga. Golola: Golola B and Kibaka were also randomly sampled. Mothers who had had home births in the previous two years before the study or their partners were purposively sampled through the local council chairpersons of the sampled villages who used loudspeakers and word of mouth to invite them to predetermined primary schools for a health study. Mothers and fathers with the above characteristics who turned up and consented/ assented were included in the study.

The sample size was obtained by Godden formula as it is suitable for calculating sample size when the overall population in the study area is greater than 50,000 as below:

$$
N=\frac{Z^{2} p q}{d^{2}}
$$


Where:

$\mathrm{N}=$ the desired sample size

$\mathrm{Z}=$ the standard normal deviation at $95 \%$ confidence level $=1.96$

$\mathrm{p}=$ the proportion in the target population estimated to have characteristics being measured.

According to the DHO and the biostatistian Gomba district, (2013) as well as the HMIS data sheet (2010/011, 2011/012, 2012/2013) for the district as indicated in Table 1, the highest health facility births the district had ever had since 2010 was $26 \%$ of the expected births in the district. Therefore the proportion of home deliveries in Gomba was;

$100-26=74 \%=0.74$

$\mathrm{q}=1-\mathrm{p}=1-0.74=0.26$

$\mathrm{d}=$ the level of statistical significance set $=0.05$

Therefore sample size; $\mathrm{n}=\frac{1.96^{2} \times 0.74 \times 0.26}{0.05^{2}}=295.649526$

from the whole district. Therefore, since only two out of five sub-counties were included in the study, the specific actual sample size for mothers was;

\section{2 $\frac{2}{5} \times 296=118$ (Including both mothers and fathers who represented their wives) 5}

In addition to the above sample, 2 health center administrators and 4 midwives were also included as key informants, making a total of 124 participants.

\section{Data Collection}

An introductory letter and ethical clearance were obtained from the Uganda Christian University Research Committee; Protocol (RJ11M21/068). These were presented to relevant authorities in the study area. Having acquired permission from District Health Officer, 2013 and sought written informed consent and assent from participants, a pretested semi-structured questionnaire and focus group discussion guide were used to collect data from the mothers and their sexual partners while a key informant interview guide was used to collect data from health facility administrators and midwives. Each interview took 20 minutes while each focus group discussion composed of 8 to 10 participants lasted for 30 minutes. The whole process of data collection took a period of one month.

\section{Statistical Analysis}

Quantitative data were coded and entered into statistical software called Statistical Package for Social Scientists (SPSS) version 16. Analysis of descriptive statistics and association of the mothers' social demographic characteristics with their choice of birthplace in this case, the home was determined at 0.05 level of statistical significance. Qualitative data was thematically analyzed by identifying major themes which were placed together for abstraction and direct quotations were made in some instances.

\section{Results}

Both qualitative and quantitative data were presented simultaneously as below. Although 124 participants were expected, 133 participants turned up and consented/ assented for the quantitative method in addition to 2 health center administrators and 4 midwives, for the qualitative method, making a total of 139 participants.

\section{Socio-Demographic Characteristics of the Mothers}

The socio-demographic characteristics of the mothers that were measured included age, marital status, level of family income, tribe, religion, mother's occupation, parity, and mother's and father's levels of education, culture, distance from home to the health center, transport network and availability of a caregiver to stay with other children at home and or to accompany the expectant mother to the health center at the time of birth. These are shown in Table 2 as explained below: 


\begin{tabular}{|c|c|c|c|}
\hline Variable & Category & Frequency & Percentage \\
\hline \multicolumn{4}{|c|}{ Age } \\
\hline & Below 25 & 18 & 13.53 \\
\hline & $25-34$ & 62 & 46.62 \\
\hline & 35 and above & 53 & 39.85 \\
\hline \multicolumn{4}{|c|}{ Religion } \\
\hline & Anglican & 39 & 29.32 \\
\hline & Catholic & 36 & 27.07 \\
\hline & Muslim & 48 & 36.09 \\
\hline & Others & 10 & 7.52 \\
\hline \multicolumn{4}{|c|}{ Tribe } \\
\hline & Muganda & 115 & 86.47 \\
\hline & Others & 18 & 13.53 \\
\hline \multicolumn{4}{|c|}{ Marital status } \\
\hline & Single & 24 & 18.05 \\
\hline & Married & 98 & 75.68 \\
\hline & Others & 11 & 8.27 \\
\hline \multicolumn{4}{|c|}{ Number of children born from home } \\
\hline & 1 Child & 1 Child & 39.85 \\
\hline & 2 Children & 2 Children & 39.85 \\
\hline & 3 Children & 3 Children & 14.29 \\
\hline & More than 3 & 8 & 6.02 \\
\hline \multicolumn{4}{|c|}{ Parity } \\
\hline & Primipara & 11 & 8.27 \\
\hline & Multipara & 102 & 76.69 \\
\hline & Grand Multipara & 20 & 15.04 \\
\hline \multicolumn{4}{|c|}{ Employment Status } \\
\hline & $\begin{array}{c}\text { Formal } \\
\text { employment }\end{array}$ & 7 & 5.26 \\
\hline & Self-employment & 62 & 46.62 \\
\hline & House wife & 40 & 30.08 \\
\hline & $\begin{array}{c}\text { Informal } \\
\text { employment }\end{array}$ & 24 & 18.05 \\
\hline \multicolumn{4}{|c|}{ Mother's level of education } \\
\hline & None & 65 & 48.87 \\
\hline & Primary & 52 & 37.10 \\
\hline & $\begin{array}{l}\text { Secondary and } \\
\text { above }\end{array}$ & 16 & 12.03 \\
\hline \multicolumn{4}{|c|}{ Father's level of education } \\
\hline & None & 36 & 27.07 \\
\hline & Primary & 64 & 48.12 \\
\hline & $\begin{array}{l}\text { Secondary and } \\
\text { above }\end{array}$ & 33 & 24.81 \\
\hline Total & & 133 & 100.00 \\
\hline
\end{tabular}

Table 2: Social Demographic Characteristics of the Mothers who had Home Birth(s)

Among the 133 participants who had had home births, 18 (13.53\%) were below the age of 25 years, 62 (46.62\%) were between 25 to 34 years and $53(39.85 \%)$ were 35 years and above. the study found that mothers representing various religions in the area of study had relatively equal chances of giving birth at home. Of the total participants, the Anglicans were 39 (29.32\%), Catholics were 36 (27.07\%), Muslims were 48 (36\%) and the other religions were 10 (7.52\%). Besides, 115 (86.47\%) of them were of Baganda tribe, and $18(13.53 \%)$ represented all other tribes. This does not necessarily mean that home birth is more prevalent among the Baganda but it is because they are the traditional inhabitants of the area.

Marital status included; singles, married (in church and cohabiting), divorced, separated and widowed. It was found out that, 98 (75\%) of the participants were married, 24 (18.05\%) were single while $11(8.27 \%)$ were divorced, separated or widowed. This 
indicated that home births were more prevalent among married mothers. The study also reported that the number of mothers who had had either one or two children from home was more than that of the grand multipara mother. This could be explained by the finding of age where fewer younger mothers gave birth at home than middle-aged mothers indicating that most of them had their first child in health facilities and had successive births at home. It was found out that the majority of the mothers who had had home births 102 (76.69\%) of them were multipara meaning that, they had already had at least a child. Grand multipara mothers were 20 (15.04\%) while Primipara mothers were 11(8.27\%).

Self-employed mothers (farmers and business mothers) were the majority of those that had had home births followed by housewives, mothers in the informal sector, and lastly by mothers in the formal sector. Self-employed mothers were 62 (46.62\%), house wives were 40 (30.08\%), those under informal employment were 24 (18.05\%), and those who were formally employed were 7 (5.26\%). The mothers who had not attained any level of education were 65 (48.87\%); those with primary level were 52 (37.10\%) while those of secondary and above were 16 (12.03\%). Most of the mothers who had had home birth had sexual partners with primary or no level of education. Table 2 also indicates that $64(48.12 \%)$ of the fathers had a primary level of education, $36(27.07 \%)$ had no education at all while only $33(24.81 \%)$ had a secondary level and above.

Much as all the factors above influenced home births to a certain extent, mothers' age, level of education, fathers' levels of education and distance from the health facility were emphasized by all FDGs and key informants. This indicates that they have a greater influence on home births than the rest of the determinants. "Some mothers attend antenatal care but they do not come to deliver here. To mothers who are delivering the first child, it is cultural to deliver at the in-laws' home rather than at the health facility" while to others, it may be due to the long distance to cover yet transport means are difficult in this area. To others, it may be due to failure to plan and decide where to give birth from and also the sudden onset of labor". A midwife at one of the health centers in Kabulasoke sub-county narrated.

Table 3 represents a bivariate analysis of the association of social demographic characteristics and their ultimate choice of place of birth which was home in this study. Findings indicated a significant association between age and the mothers' choice of place of birth with a chi-square $\left(\mathrm{X}^{2}\right)$ of 9.5006 and P-value of 0.009 at an alpha level of 0.05 . Marital status and the choice of place of birth at $\mathrm{X}^{2}=7.4953$ and $\mathrm{P}$-value was 0.02 , and the previous number of births already had at home at $\mathrm{X}^{2}=10.0139$, and $\mathrm{P}$-value $=0.018$. Presence or absence of someone to assist in the process of labor and childbirth was also significantly associated with the choice of birthplace at $\mathrm{X}^{2}=12.5205$ with a P-value $=0.002$. Parity was also statistically associated at $\mathrm{X}^{2}$ of 9.9709 , P-value of 0.007 . Other factors that were significantly associated included the mother's employment status, mother and the father's levels of education, and the distance between home and health facility. The characteristics of the health centers indicated no association with the choice of place of birth at $\mathrm{X}^{2}=1.2654$, and $\mathrm{P}$-value $=0.737$. There was also no association with religion at X2 $=1.864 ; \mathrm{P}$-value $=$ 0.601 , and tribes at $\mathrm{X}^{2}=0.6345$; P-value $=0.426$ at alpha level of 0.05 .

\begin{tabular}{|c|c|c|c|c|}
\hline Variable & Home birth (\%) & Health unit (\%) & & \\
\hline \multicolumn{4}{|c|}{ Age } & \\
\hline Below 25 & 38.89 & & & \\
\hline $25-34$ & 70.97 & & $\mathrm{X}^{2}=9.5006$ & $\mathrm{P}$-Value $=0.009$ \\
\hline Above 34 & 77.39 & 22.64 & & \\
\hline \multicolumn{4}{|c|}{ Religion } & \\
\hline Anglican & 69.23 & 30.77 & & \\
\hline Catholic & 61.11 & 38.89 & & \\
\hline Muslim & 75.00 & 25.00 & $\mathrm{X}^{2}=1.8648$ & $\mathrm{P}-$ Value $=0.601$ \\
\hline Others & 70.00 & 30.00 & & \\
\hline \multicolumn{4}{|c|}{ Tribe } & \\
\hline Muganda & 70.43 & 29.57 & $\mathrm{X}^{2}=0.6345$ & P-Value $=0.426$ \\
\hline Others & 61.11 & \multicolumn{2}{|c|}{38.89} & \\
\hline \multicolumn{4}{|c|}{ Number of children born at home } & \\
\hline 1 & 54.72 & 45.28 & & \\
\hline 2 & 81.13 & 18.87 & $\mathrm{X}^{2}=10.0139$ & P-Value $=0.018$ \\
\hline 3 & 68.42 & 31.58 & & \\
\hline More than 3 & 87.50 & 12.50 & & \\
\hline \multicolumn{4}{|c|}{ Assistance during labor } & \\
\hline Self & 30.00 & 70.00 & & \\
\hline Relative & 76.47 & 23.53 & $\mathrm{X}^{2}=12.5205$ & $\mathrm{P}$ - Value $=0.002$ \\
\hline TBA & 52.38 & 47.62 & & \\
\hline
\end{tabular}




\begin{tabular}{|c|c|c|c|c|}
\hline \multicolumn{4}{|c|}{ Parity } & \\
\hline Primipara & 27.27 & 72.73 & & \\
\hline Multipara & 73.53 & 26.47 & $X^{2}=9.9707$ & P- Value $=0.007$ \\
\hline Grand Multipara & 70.00 & 30.00 & & \\
\hline \multicolumn{4}{|c|}{ Employment Status } & \\
\hline Formal employment & 00.00 & 100.00 & & \\
\hline Self employed & 72.58 & 27.42 & & \\
\hline House wife & 77.50 & 22.50 & $\mathrm{X}^{2}=17.4163$ & P-Value $=0.001$ \\
\hline Informal employment & 66.67 & 33.33 & & \\
\hline \multicolumn{5}{|c|}{ Factors affecting choice of birthplace } \\
\hline Attitude of midwives & 41.67 & 58.33 & & \\
\hline Long distance & 79.22 & 20.78 & $\mathrm{X}^{2}=10.2601$ & P-Value $=0.016$ \\
\hline Lack of money & 63.16 & 36.84 & & \\
\hline Others & 56.00 & 44.00 & & \\
\hline \multicolumn{4}{|c|}{ Mother's level of education } & \\
\hline None & 87.69 & 12.31 & & \\
\hline primary & 67.31 & 32.69 & $\mathrm{X}^{2}=46.4416$ & P-Value $=0.000$ \\
\hline Secondary and above & 0.00 & 100.00 & & \\
\hline \multicolumn{5}{|c|}{ Father's level of education } \\
\hline None & 91.67 & 8.33 & & \\
\hline primary & 92.19 & 7.81 & $\mathrm{X}^{2}=98.4878$ & P-Value $=0.000$ \\
\hline Secondary and above & 0.00 & 100.00 & & \\
\hline \multicolumn{5}{|c|}{ Distance from home to health unit } \\
\hline $0-3 \mathrm{Km}$ & 60.00 & 40.00 & & \\
\hline $3-6 \mathrm{Km}$ & 48.48 & 51.52 & $\mathrm{X}^{2}=10.9278$ & P- Value $=0.004$ \\
\hline$>6 \mathrm{Km}$ & 78.82 & 21.18 & & \\
\hline \multicolumn{5}{|c|}{ Marital status } \\
\hline Single & 45.83 & 54.17 & & \\
\hline Married & 74.49 & 25.51 & $\mathrm{X}^{2}=7.4953$ & $\mathrm{P}$ - Value $=0.02$ \\
\hline Others & 72.73 & 27.27 & & \\
\hline \multicolumn{5}{|c|}{ Characteristics of health units } \\
\hline $\begin{array}{l}\text { Corruption among } \\
\text { health workers }\end{array}$ & 69.05 & 30.95 & & \\
\hline Attitude of midwives & 72.22 & 27.78 & $\mathrm{X}^{2}=1.2654$ & P- Value $=0.737$ \\
\hline Fear of baby exchange & 62.50 & 37.50 & & \\
\hline Others & 55.56 & 44.44 & & \\
\hline
\end{tabular}

Table 3: Bivariate Analysis of Independent Variables against Dependent Variable

\section{Role of VHTs in Referring Mothers for Health Facility Birth}

All the villages in Gomba district had VHTs by the time the mothers had a home birth(s). VHTs had been in their villages for more than five years but they had to a low extent advised mothers to attend ANC. Even then, they did not highlight the dangers of giving birth at home. "They are mainly involved in disease prevention strategies including education on the use of treated mosquito nets and their distribution. If they were really engaged in referring mothers for health facility birth, we would have already registered a difference in facility birth but it has been relatively constant". Said an in charge of a health center. Two of the focus groups had the same perspective.

\section{Influence of Partners on Home Births}

Key informants in all the sampled health centers and focus group discussions stated that males were rarely involved in maternal and reproductive health Issues such as ANC and Prevention of Mother to Child Transmission (PMTCT) of HIV but had an upper hand in deciding the choice of birthplace more especially for the married couple. From quantitative data findings, more married women had had home births than single and separated women. Marital status was also found to be statistically associated with home birth at $\mathrm{X}^{2}=7.4953$ and P-value of 0.02 at $\alpha=0.05$. The key informants reported that fathers determined the choice of place as heads of families and because it had financial implications. One key informant stated that "for married couples, the mothers 
relied on the spouse's choice of place of birth because they are the ones to eventually pay hospital charges. For single mothers, I highly doubt if their partners can influence the choice of birthplace since they are not staying together". However, FDG 5 mentioned that "The mothers proposed and the fathers agreed to the mother's choice". Also, the single mothers decided for themselves because in most cases the fathers were not supportive.

\section{Discussion}

\section{Socio-Demographic Characteristics of the Mothers}

The study found out that socio-demographic characteristics such as age, marital status, mother's and father's level of education, distance between home and health facility, family income and lack of a caregiver to leave at home or accompany the mother in the health facility were associated with home birth except for religion, tribe, and health facility characteristics. Culture determined the choice of birthplace for the first child among the Baganda mothers. Though Gwamaka S, had similar findings on the subject, he noted that religion influenced the choice of birthplace but did not state whether the choice was in favor of home birth or health facility [23].

Findings by Kkonde A and Moyer AC, Mustafa A, also noted that religion, marital status, and the respondent's level of education influenced home births [24,25]. As far as religion is concerned, mainly traditionalists and Muslims in certain countries are more likely to deliver at home. Young mothers aged 20 to 25 years and elderly mothers aged 38 to 43 years planned and had a home birth than middle-aged ones [24]. Studies by Uganda Bureau of Statistics, Kkonde A and Moyer AC, Mustafa A also noted that younger women of reproductive age delivered under the care of SBAs than older women which correspond with the findings of this study $[21,24,25]$. However, those who are much younger, especially below eighteen years, are less likely to have health facility birth [21]. Other factors include culture, personal preferences and unplanned home births due to the sudden onset of labor $[25,26]$.

According to the World Health Organisation (WHO), poor socioeconomic determinants of health greatly contribute to home birth for example, wealthy women are three folds more likely to deliver in a health facility than poor women. The same applies to $75 \%$ of highly educated women compared to $25 \%$ of less educated women [5]. In contrast with findings of this study and those revealed by WHO, are findings by De Allegri M, Tiendrebéogo J, Müller O, et al., indicated no significant relationship between lower socioeconomic status and home birth [5,27]. It also indicated no significant trend between residential areas and home birth although it found a positive relationship between distance to the health facility and home delivery. It pointed out two major barriers to health services delivery as geographical access in terms of road condition high transport fares, and higher user fees. In some communities though, even when roads are in good condition, health facilities well facilitated and user fees waived, home birth remains a normal event. For that reason, health facility birth is a last resort following complications [11]. This is attributed to the inadequacy of skilled staff at lower health center levels, abuse by health workers through neglect and or poor treatment [11]. The three delays imbedded in gender inequality and poor past experiences with health workers, and inadequate quality of health services generally influence home birth [19].

As a result, TBAs continue to operate in several rural communities in Sub-Saharan Africa including Uganda, assisting a wide number of women in making choice of birthplace, and in child delivery despite increased emphasis on SBA $[8,28]$. This has contributed to high maternal death of about 16 women per day, 94 stillborn babies, and 81 neonates' death on a daily basis [6].

\section{Role of VHTs in Referring Mothers for Health Facility Birth}

VHTs have not done much in referring mothers for health facility birth in Gomba district but are rather actively involved in the prophylaxis of malaria, mobilization of masses for immunization, and prevention of diarrheal diseases. A study done in Northern Uganda by Rudrum S, indicated that they are also involved in disseminating health information, coordinate outreach programmes in liaison with formal health workers, and deliver essential drugs to households [8]. A study carried out in Tanzania by Mushi D, Mpembeni R, Jahn A, reported that actively involving VHT in maternal and child health had increased health facility deliveries from $34.1 \%$ to $51.4 \%(\mathrm{P}<0.005)$ [29].

The VHTs in Tanzania were found to have a number of roles among which include referring expectant mothers for health facility birth [29]. However, according to Ntalo, little has done in Uganda due to inadequacy in drawing birth plans which VHTs would use to sensitize mothers [30]. However, by 2015 a training manual in maternal and child health had been designed specifically for VHTs. The manual highlighted VHTs key messages to mothers and their roles in improving maternal and child health. Among other roles include: registering all pregnant women in their villages as well as their under-five children, providing health education and promotion, help communities to identify, understand and be able to solve their health problems, carrying out regular home visits during pregnancy and soon after birth, supporting and mobilizing communities for action planning, transportation planning as well as child health days [30]. On the other hand, WHO attributes home births to challenges within health systems including less motivated health workers, shortage of trained health workers, inadequate logistics, inequitable interventions and inadequate essential drugs [5].

\section{Influence of Partners on Home Births}

Partners were found to influence the choice of birth place. Most married women had had home births than single and separated 
women, an indication that partners influenced the choice of birth place. However, according to Banyana $\mathrm{C}$ partners had no influence on home births [26]. The same study points out that some mothers who were married reported that their partners did not take the initiative to inquire about where they intend to give birth from while a study by Banyana $\mathrm{C}$ found out that mothers made their final decision on birth place although they consulted their husbands or other people [26]. It also pointed out that some mothers did not consult anyone but did so if complications arose. A study by Abeje G, Azage M, Setegn T, also notes the husbands' socio-demographic and economic factors greatly influence the choice of delivery place as well as general utilisation of health services [4]. Further research should be done to investigate why the birth of a first child is preferred in a home setting and mainly at the in-laws' place of residence among some Baganda women.

While findings of this study emphasize cultural values to influence home birth especially partners, a study in Burkina Faso by Moyer AC, Mustafa A noted that mothers and their households valued facility-based delivery over and above home delivery [25]. This could be due to cultural diversity in African settings.

\section{Conclusion}

Conclusively, bridging the distance between homes and health facilities as well as empowering the girl child through education would reduce home births. VHTs and partners should be lobbied to actively engage in maternal and childbirth. Also, multipartner initiatives are desired in rural communities to address the issue of homebirths.

\section{Acknowledgement}

The author extends heartfelt gratitude to Dr. Edward Mukooza; her research supervisor, Dr. Edward Kanyesigye; the Dean of Faculty of Technology and Health Sciences and Dr. Ekie Kikule; the Head of Department of Health Sciences, Uganda Christian University for their continuous support as regards the course undertakings. Sincere gratitude goes to Taremwa Danison, Ignatius Abaijuka, Nandora Christine, Asiimwe James, Selah Agaba, Mugisha Charles, Sophia Nakanjako, Kaitetsi Catherine, Ndinawe Medard and Atimu Betty for their strong support during this project deveopment. Lastly but not least, heartfelt appreciation to the research assistants; Namuddu Teddy, Lubuga Juliana, Joseph Sserungaya, Phiona Namwase, and Kabuuka Jamil, to the mothers, fathers, local council chairpersons, midwives and the district health team of Gomba district who participated in the study by providing invaluable information making it a success.

\section{References}

1. Wesson N (2006) Homebirth A practical guide. Pinter \& Martin Publishers, UK.

2. ACNM (2011) Position statement, Home birth, American College of Nurse-Midwives.

3. UNFPA (2017) Population matters. Midwifery services in Uganda, United Nations Population Fund, Uganda.

4. Abeje G, Azage M, Setegn T (2014) Factors associated with Institutional delivery service utilization among mothers in Bahir Dar City administration, Amhara region: a community based cross-sectional study. Reprod Health 11: 22.

5. WHO (2011) The partnership for maternal, newborn and child health, Maternal and Child Health, Uganda.

6. Kigozi S (2017) Childbirth in Uganda: Stories from women and health workers, Save the Children.

7. Shallon A, Ojengbede AO, Mugisha JF, Odukogbe AA (2018) Social reintegration and rehabilitation of obstetric fistula patients before and after repair in SubSaharan Africa: A Systematic Review. Nepal J Obstetrics Gynecol 24: 5-14.

8. Rudrum S (2015) Traditional Birth Attendants in Rural Northern Uganda: Policy, Practice, and Ethics. Health Care Women Int 37: 250-69.

9. Grieco M, Turner J (2005) Maternal mortality Africa's burden Toolkit on gender, transport, and maternal mortality, Transport Research Institute, Napier University.

10. Serbanescu F, Goldberg H, Daniel I, Wuhib T, Marum L (2017) Rapid reduction of maternal mortality in Uganda through the Saving Mothers, Giving Life Initiative: Results of 1-year evaluation. BMC Pregnancy and Childbirth 17:42.

11. Kyomuhendo BG (2003) Low use of rural maternity services in Uganda: Impact of women's status, traditional beliefs, and limited resources. Reprod Health Matters 11: 16-26.

12. Degefie T, Amare Y, Mulligan B (2014) Local understandings of care during delivery and postnatal period to inform home based package of newborn care interventions in rural Ethiopia: a qualitative study. BMC Int Health Human Rights 14: 17.

13. Prata N, Mbaruku G, Campbell M, Potts M, Vahidnia F (2005) Controlling Postpartum haemorrhage after home birth in Tanzania. Int J Gynecol Obstet 90: 51-5. 14. WHO (2018) Country cooperative strategy at a glance: Uganda. World Health Organization.

15. The Republic of Uganda (2015) Roadmap for accelerating the reduction of maternal and neonatal mortality and morbidity in Uganda 2007-2015, Republic of Uganda.

16. Uganda Bureau of Statistics (UBOS) Uganda Demographic and Health Survey 2011 (UDHS), MEASURE DHS ICF International, Calverton, Maryland, USA.

17. Morrison J, Thapa R, Basnet M, Budhathoki B, Tumbahangphe K (2014) Exploring the first delay: a qualitative study of home deliveries in Makwanpur district Nepal. BMC Pregnancy Childbirth14: 89.

18. Ministry of Health (2006) Health Sector Strategic and Investments Plan for Uganda (2001/2002- 2005/2006). MOH, Uganda.

19. Kananura R (2012) Role of Village Health Teams (VHTs) in Public Health-Uganda. American Public Health Association, Apha 140th Annual Meeting and Expo, San Francisco, CA.

20. Healthy Child Uganda (2015) Village Health Team Maternal Newborn and Child Health Training Manual. Mbarara University of Science and Technology, Mbarara, Uganda.

21. Uganda Bureau of Statistics (UBOS) (2012) Statistical abstract 2012. Uganda Bur Stat 1-228. 
22. Uganda Bureau of Statistics (2016) National Population and housing Census 2014. Uganda Bur Stat 1-105.

23. Gwamaka S (2012) Utilization and factors affecting delivery in health facilities among recently delivered women in Nkasi district, Muhimbili University of Health and Allied Sciences, Master of Public Health Dissertation.

24. Kkonde A (2010) Factors that Influence pregnant women's choice of delivery site in Mukono district- Uganda, University of South Africa.

25. Moyer AC, Mustafa A (2013) Drivers and deterrents of facility delivery in sub-Saharan Africa: a systematic review. Reprod Health10: 40.

26. Banyana C (2001) Women's decision-making and factors affecting their choice of place of delivery: Systematic review and qualitative study. Surrey Res Insights 13: 59.

27. De Allegri M, Tiendrebéogo J, Müller O, Yé M, Jahn A, et al. (2015) Understanding home delivery in a context of user fee reduction: a cross-sectional mixed methods study in rural Burkina Faso. BMC Pregnancy and Childbirth 15: 330.

28. Kabakyenga JK, Ostergren PO, Turyakira E, Pettersson KO (2012) Influence of birth preparedness, decision-making on location of birth and assistance by skilled birth attendants among women in South-Western Uganda. PLOS One 7: e35747.

29. Mushi D, Mpembeni R, Jahn A (2010) Effectiveness of community-based safe motherhood promoters in improving the utilization of obstetric care. The case of Mtwara rural district; Tanzania. BMC Pregnancy and Childbirth 10: 14

30. Ntalo J (2012) Improve Maternal Health with VHT Training in Birth Planning.

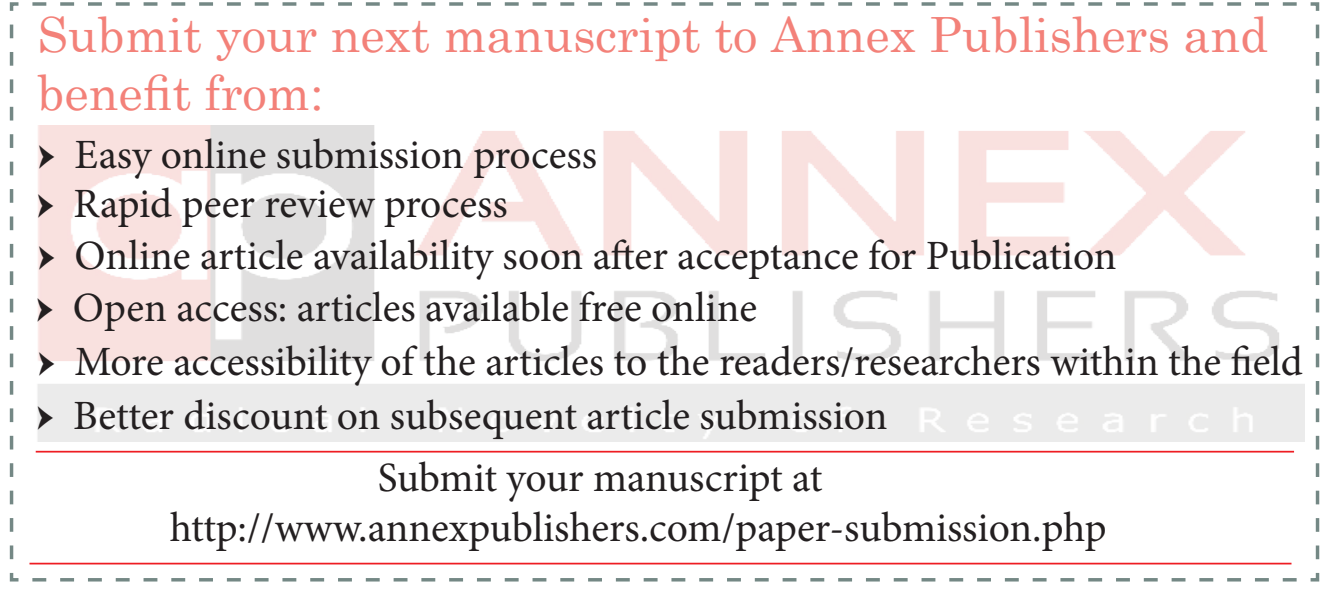

